\title{
Excimers in Multichromophoric Assemblies: Boon or Bane?
}

\author{
Debangshu Chaudhuri*a(D) \\ a Department of Chemical Sciences, Indian Institute \\ of Science Education and Research (IISER) Kolkata, \\ Mohanpur 741246, India. \\ dchaudhuri@iiserkol.ac.in
}

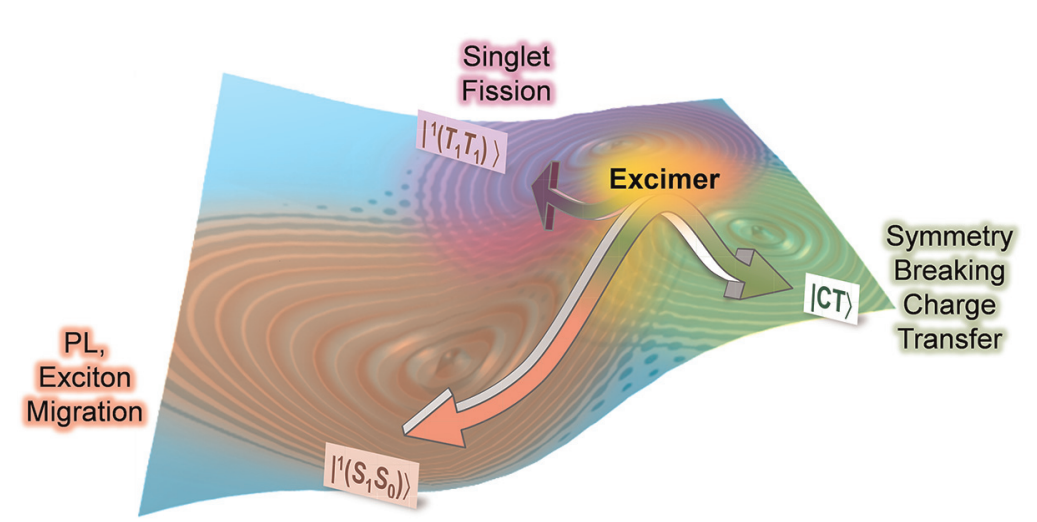

Received: 23.06.2021

Accepted after revision: 03.08.2021

DOI: 10.1055/a-1578-0960; Art ID: OM-2021-06-0036-SR

\section{License terms: $@(1)(\Theta)$}

(c) 2021. The Author(s). This is an open access article published by Thieme under the terms of the Creative Commons Attribution-NonDerivative-NonCommercial License, permitting copying and reproduction so long as the original work is given appropriate credit. Contents may not be used for commercial purposes, or adapted, remixed, transformed or built upon. (https://creativecommons.org/licenses/by-nc-nd/4.0/)

Abstract Exciton dynamics in organic semiconductors is a subject of great significance from the standpoint of light emission, as well as light harvesting. As transient excited state species, excimers are expected to play a significant role in the dynamics and the fate of the excited state. Till recently, the discourse on excimers in organic systems revolved around their role in aggregation-induced fluorescence quenching, or utilizing their characteristic red-shifted emission to report local interactions. But in the last decade, research in the area of organic multichromophoric systems has brought the spotlight back on this fascinating species. This review focuses on recent developments that highlight the importance of excimers in various processes involving multichromophoric systems, such as circularly polarized emission, exciton migration, and singlet fission. The review also attempts to address the question of whether excimers are useful or detrimental to various photophysical and photochemical processes of importance.

Table of content:

Introduction

Excimers in Multichromophoric Assemblies

Excimer Luminescence

Excimers in Light Harvesting

Conclusions and Outlook

Key words: excimers, multichromophoric assembly, circularly polarized luminescence, exciton migration, singlet fission, symmetry-breaking charge separation

\section{Introduction}

In 1941, Szent Györgyi wrote a thought-provoking article titled "Towards a New Biochemistry", 1 in which he argued that a greater number of molecules may join to form such energy continua, along which energy, viz., excited electrons, may travel a certain distance. It was one of the earliest propositions in biochemistry that a collection of molecules could behave differently from its constituents, and give rise to a new function. This very idea has also been at the heart of organic semiconductors and molecular electronics research. Over the years, the scope of organic semiconductors has considerably widened to include a variety of materials, from molecular crystals, ${ }^{2}$ thin films, ${ }^{3}$ conjugated polymers, ${ }^{4}$ dendrimers ${ }^{5}$ and more recently to multichromophoric assemblies. ${ }^{6}$ From what once was a niche research area in physics, the field now attracts organic chemists, materials scientists, device physicists, computational chemists and theoretical physicists alike, all driven by the prospect of discovering new phenomena and designing better materials. The commercialization of first organic light-emitting diode-based displays at the turn of 21st century heralded a triumph for this versatile class of materials. Since then organic semiconductors have steadily increased its footprint in photovoltaics, printed electronics, battery technology etc.

The nature of light-matter interaction and low-lying excitations is of great significance in organic semiconductors. ${ }^{7}$ Processes such as light emission ${ }^{8}$ and light harvesting ${ }^{9}$ depend on the nature of excited state and its dynamics. Its understanding is therefore critical to improving material performance in various optical and optoelectronic applications. While our understanding of light-matter interaction is largely shaped by traditional inorganic semiconductors, there are key distinctions between inorganic and organic systems. The latter is usually characterized by a strong intra- 


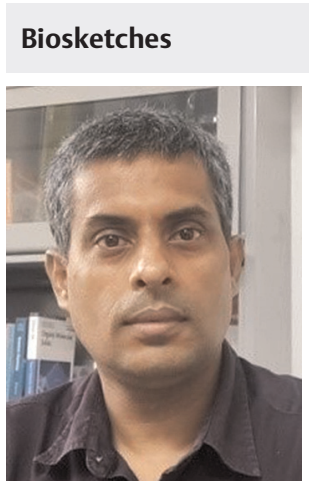

molecular and a much weaker intermolecular electronic coupling. ${ }^{10}$ This along with the fact that organic solids also have low dielectric constants ensures that the photoexcited electron-hole pair remains strongly correlated and fairly localized, spread over only a few molecular units. Such excited states are referred to as the Frenkel excitons. A consequence of this localized molecular nature of the excited state is reflected in the optical absorption spectrum of an organic semiconductor, which retains a strong resemblance to that of the free molecule. Localization of Frenkel exciton has one other important consequence: electronic transitions can couple strongly with molecular or lattice vibrations. ${ }^{11}$ Since the lowest energy transitions in organic semiconductors are mostly $\pi-\pi^{*}$ or $n-\pi^{*}$ in nature, electronic reorganization following an optical transition is likely to affect the $\pi$ bond order and therefore the underlying structure. Such strong coupling of an electronic transition to a vibrational mode increases the effective mass of the exciton, and in turn affects its mobility through the semiconductor layer. ${ }^{12}$

A localized photoexcitation can also influence the nature of van der Waals and other noncovalent interactions between molecules. A common manifestation of this is the formation of excimers. ${ }^{13}$ As a transient dimeric species that exists only in the excited state but is dissociative in the ground state, excimers are often characterized by a structureless photoluminescence (PL) spectrum that is strongly redshifted compared to that of the isolated molecule. In organic systems, excimers were first reported by Förster in concentrated solutions of pyrene. ${ }^{14}$ Over the years, excimers have been observed in thin films, molecular crystals, and more recently in multichromophoric systems like molecular clusters, ${ }^{15}$ conjugated polymers, ${ }^{16}$ and dye assemblies. In the early days, seminal works of Förster, Birks, McGlynn, Stevens and others contributed significantly towards understanding the nature of molecular interactions in excimers, ${ }^{17}$ and their formation and dissociation kinetics. ${ }^{18}$ However, a few major developments in the last 20 years have rekindled the interest in excimers. The advent of ultrafast lasers made it possible to study details of exciton dynamics that were previously inaccessible to experiments. Simultaneously, development of theoretical tools and computational power has enabled accurate modelling of excited state behaviour in complex systems. These combined with the discovery of fascinating material systems, such as conjugated polymers and dye assemblies, have expanded the scope of investigation immensely. In this review, we aim to chronicle the important developments from the last decade that present a fresh perspective on excimers, and discuss their role in light emission and light harvesting. In doing so, we restrict ourselves to a specific class of multichromophoric systems, namely the dye assemblies and related model systems.

\section{Excimers in Multichromophoric Assemblies}

The term excimer was introduced by Stevens and Hutton, ${ }^{19}$ who described it as a dimer that is associated in the excited state, but dissociates in the ground state. This definition was subsequently revised by Birks to redefine the ground state as dissociative, ${ }^{13}$ thus indicating the tendency of the bound excited pair to dissociate upon recombination, in absence of any external restraint. Different electronic interactions are possible between a pair of closely interacting excited- $\left(M^{*}\right)$ and ground-state $(M)$ molecules. Excimers are predominantly characterized by two interactions: exciton coupling and charge resonance. For the purpose of this review, we restrict ourselves to only singlet excimers. An exciton-coupled state, $\left|{ }^{1}\left(S_{1} S_{0}\right)\right\rangle$ represents the exchange of excitation energy between two identical chromophores, such that the probability of being excited is equal for both chromophores.

$\left|{ }^{1}\left(S_{1} S_{0}\right)\right\rangle=\frac{1}{\sqrt{2}}\left[\left|M M^{*}\right\rangle \pm\left|M^{*} M\right\rangle\right]$

Likewise, a charge-resonance state, $|C R\rangle$, indicates the possibility of a symmetric charge transfer between the molecules.

$|\mathrm{CR}\rangle=\frac{1}{\sqrt{2}}\left[\left|M^{+} M^{-}\right\rangle \pm\left|M^{-} M^{+}\right\rangle\right]$

Individually, neither of these pure states can fully describe the excimer. Instead, its mixed character is best captured by a coherent superposition of these states: 
$\left.\left|\psi_{\mathrm{Ex}}\right\rangle=\left.c_{1}(t)\right|^{1}\left(S_{1} S_{0}\right)\right\rangle+c_{2}(t)|\mathrm{CR}\rangle$

For the superposition or mixing to be efficient, the pure states must bear structural and energetic similarities, and a reasonably strong coupling that is comparable to their energy difference. The superposition state, $\left|\psi_{\text {Ex }}\right\rangle$ described above represents a molecular association in the excited state. Upon relaxation, it dissociates into a pair of monomers in the ground state $\left|S_{0}\right\rangle$. One realizes that the above description of excimers needs a revision before it can be applied to multichromophoric systems, like dye assemblies (Figure 1 ). In these systems, reasonably strong electronic coupling can exist between chromophores even in the ground state. Therefore, for the description of a dissociative ground state to be valid, the nuclear coordinates in the ground and the excited states of the multichromophoric system must be significantly different. The revised definition must account for the possibility that excimers in a system of multiple coupled chromophores may not be a strictly dimeric species. Further, as the size and complexity of a multichromophoric system increase, one may encounter more than one unique excimer that could interconvert during the lifetime of the excited state.

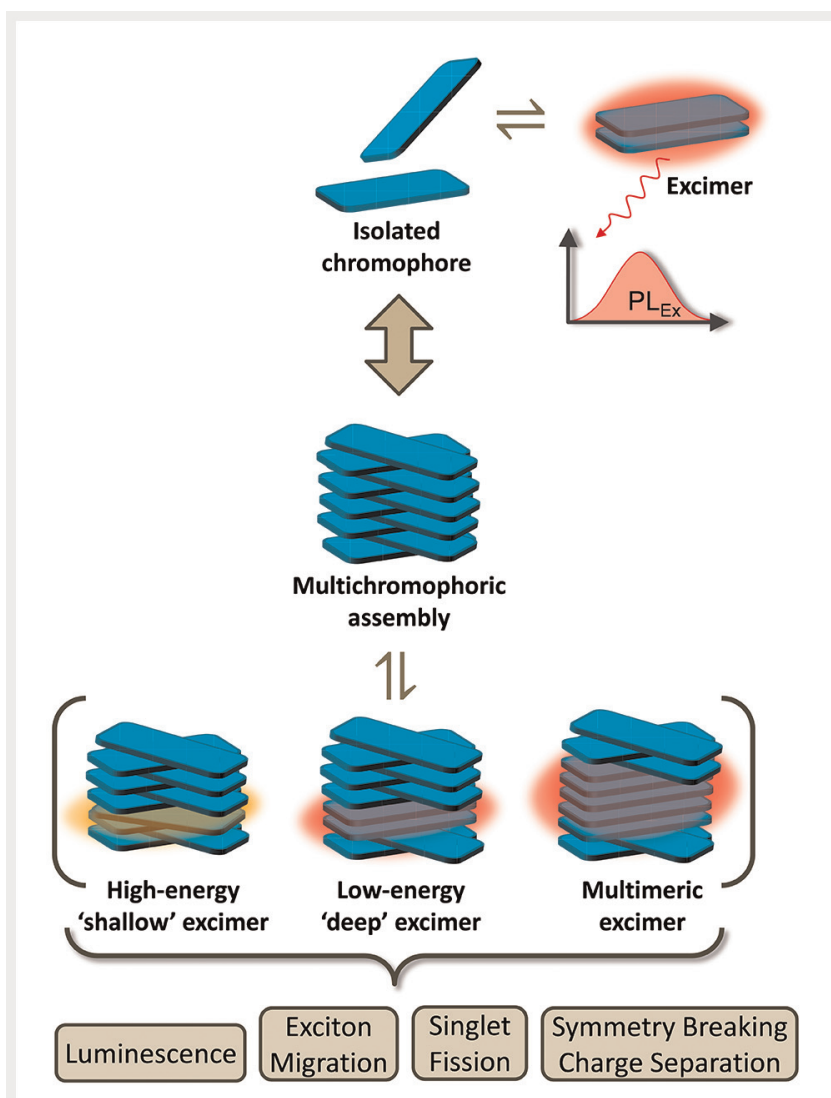

Figure 1 Excimers in multichromophoric assemblies and their role in various photophysical and photochemical processes.
In recent years, ultrafast transient absorption spectroscopy has been particularly useful in characterizing excimers. One can identify distinct spectroscopic signatures corresponding to the exciton-coupled and charge-transfer (CT) characters, with relative contributions given by their timedependent probabilities, $\left|c_{\mathrm{i}}{ }^{2}\right|$. By monitoring how the coupling between the pure states evolves over time, one can investigate relaxation pathways in excimers, and its involvement in other excited-state processes. Every molecule is different. And so are the structural changes that accompany excimer formation, and the dominant interactions that operate within such a species. While the term excimer is broadly used to indicate a transient, excited-state association, the way it manifests in various photophysical and photochemical processes can be very different in different multichromophoric systems.

\section{Excimer Luminescence}

A few notable exceptions aside, ${ }^{20}$ excimers usually suffer from low PL efficiency and a poor colour purity. These factors limit their utility as emissive materials in applications that require a bright light output. Yet, one of the more successful applications of excimers in the past relied on the appearance of its characteristic red-shifted PL to report specific molecular interactions. In particular, this approach has shown great promise in biomedical science, where the ability to detect a point mutation or a single nucleotide mismatch in a genome holds enormous significance in the early detection of genetic diseases. The strategy involves the use of synthetically modified nucleotides with carefully incorporated chromophores that upon hybridization with the complementary strand gives rise to an excimer PL. The use of excimer luminescence in DNA hybridization has been extensively reviewed by Kool et al., ${ }^{21}$ and will not be discussed here any further.

In recent times, a considerable amount of research has focussed on the possibility of getting circularly polarized luminescence (CPL) from excimers. ${ }^{22}$ CPL refers to the differential PL emission of the left $\left(I_{L}\right)$ and right $\left(I_{R}\right)$ circularly polarized light, and is quantified in terms of the luminescence dissymmetry factor $\left(g_{\text {lum }}\right)$ which is given by $g_{\text {lum }}=2\left(I_{R}-I_{L}\right) /\left(I_{R}+I_{L}\right)$. A high dissymmetry in CPL correlates directly with the chiral arrangement in the excited state. Since excimers exist only in the excited state, a dissymmetric or chiral arrangement of chromophores in the excimer state could be the key to achieving a high CPL anisotropy. The possibility of excimer CPL was first demonstrated by Brittain and Fendler using enantiomeric pyrene derivatives. ${ }^{23}$ Despite a pronounced circular dichroism, the molecular state showed no dissymmetry in the PL. The excimeric state on the other hand exhibited CPL with a large $g_{\text {lum. }}$. From a practical standpoint, achieving CPL from excimers in concentrated solutions is 
not very useful. In a significant development, Inouye et al. demonstrated the possibility of getting excimer CPL from achiral chromophores. ${ }^{24} \mathrm{~A}$ pair of stacked alkynyl-substituted pyrene molecules was first trapped within a $\gamma$-cyclodextrin cavity to form a $2: 2$ inclusion complex, ${ }^{24 a}$ which was subsequently converted into a threaded rotaxane using a capping moiety (Figure 2c). Spatial restriction of pyrene chromophores encouraged the formation of excimers that showed a high PL quantum yield ( $\varphi=3.7)$. Further, the chiral environment of the cyclodextrin cavity imposed a twisted stacking of pyrene chromophores with a well-defined chirality. This resulted in a CPL with a high $g_{\text {lum }}$ value of
$-1.5 \times 10^{-2}$ at $480 \mathrm{~nm}$. The generality of this strategy was extended to obtain excimeric CPL from a pair of perylene chromophores with $g_{\text {lum }}=-2.1 \times 10^{-2}$ at $573 \mathrm{~nm}^{24 \mathrm{~b}}$ A unique advantage of excimer-based CPL materials lies in the possibility of achieving CPL in the near-infrared (NIR) region, without necessarily using NIR dyes. This was demonstrated using an anthracene-based double helicate, ${ }^{25}$ in which two helical oligo( $p$-phenyleneethynylene) units were hinged by flexible, chiral binaphthyl moieties (Figure 2b). Structural change following the photoexcitation led to the formation of an intramolecular anthracene excimer in $\mathrm{CHCl}_{3}$ that exhibited a CPL emission with $15 \%$ quantum yield and a $g_{\text {lum }}=1.1 \times 10^{-2}$ at

\section{Chiral Chromophores}

(a)

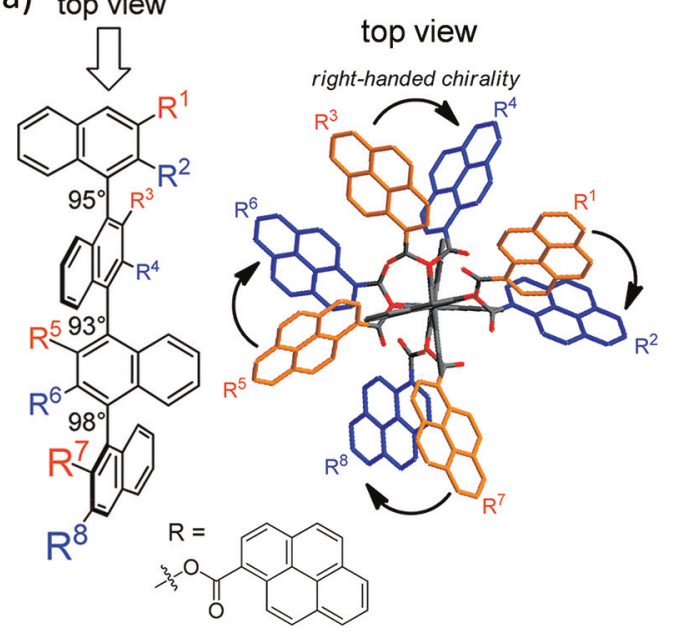

(b)
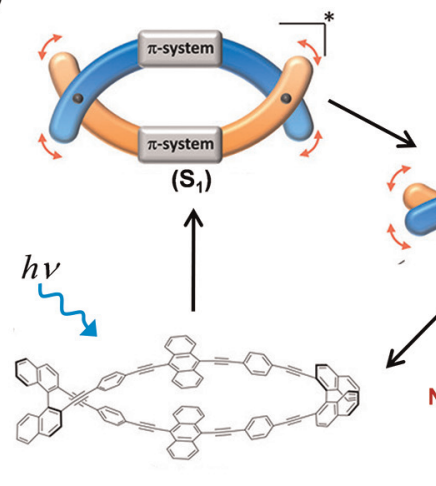

Flexible double helicate $\left(S_{0}\right)$
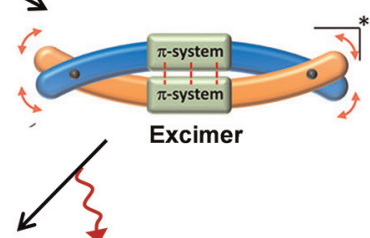

NIR CPL

\section{Achiral Chromophores}

(c)

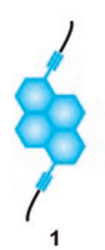

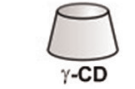

Complexation

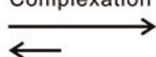

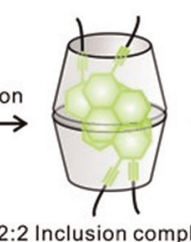

2:2 Inclusion complex

$(1)_{2} \subset(\gamma-C D)_{2}$

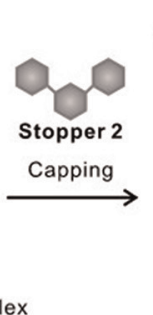

(n)

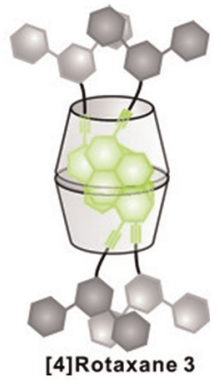

(d)
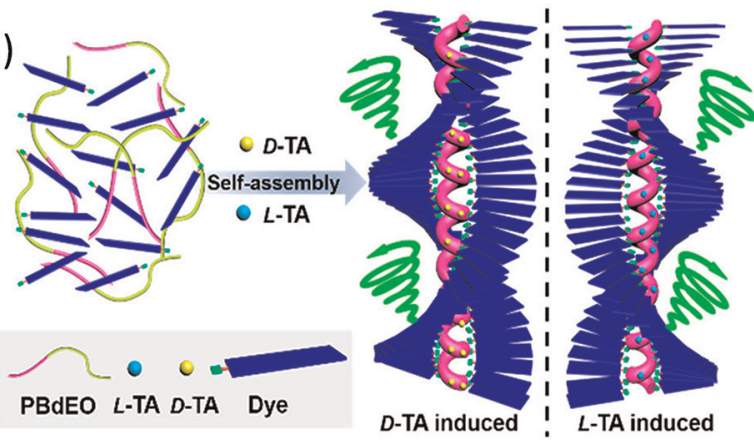

Figure 2 Achieving dissymmetry in the excimer state. Substituting multiple chromophores on a chiral backbone: (a) pyrene chromophores on a twisted quaternaphthyl scaffold interact in the excited state to generate a chiral excimer. Adapted with permission from Ref. 26a. Copyright 2018 The Royal Society of Chemistry. (b) A flexible double helicate backbone that allows two $\pi$-conjugated segments to interact closely in the excited state to create an excimer that emits CPL in the NIR region. Adapted with permission from Ref. 25. Copyright 2019 Wiley-VCH Verlag GmbH \& Co. KGaA, Weinheim. The other approach makes use of noncovalent interactions to organize achiral chromophores in a dissymmetric fashion: (c) a pair of pyrene molecules encapsulated inside a pair of y-cyclodextrins assumes a twisted stack. Adapted with permission from Ref. 24a. Copyright 2014 Wiley-VCH Verlag GmbH \& Co. KGaA, Weinheim. (d) An amphiphilic diblock copolymer PBdEO organizes helically in the presence of a chiral additive (D/L-tartaric acid), which in turn acts as a scaffold on which achiral dye molecules organize to form extended chiral assemblies. Adapted with permission from Ref. 27. Copyright 2020 American Chemical Society. 

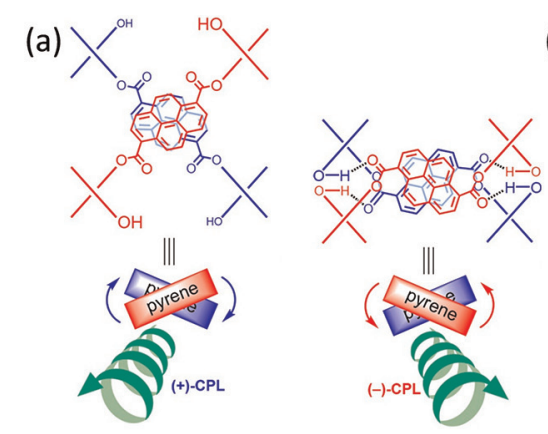

(b)
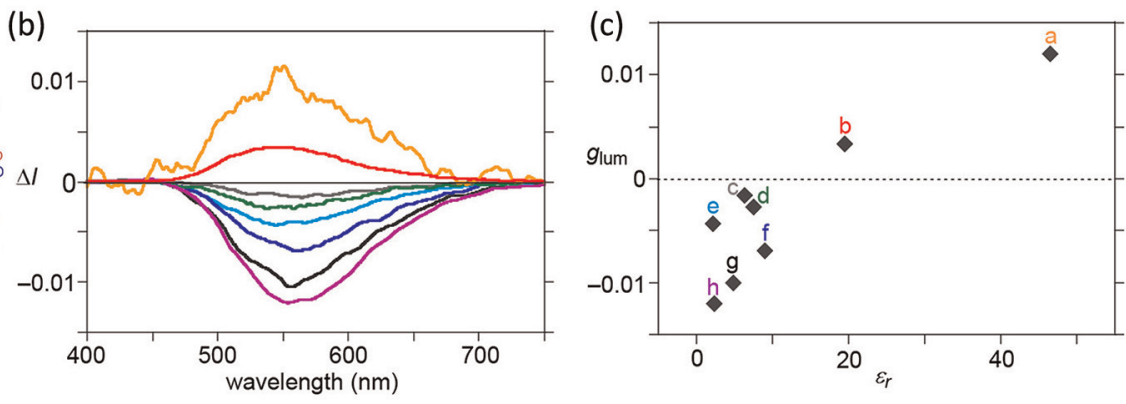

(a) DMSO (b) Acetone (c) EtOAc (d) THF (e) 1,4-dioxane (f) DCM (g) $\mathrm{CHCl}_{3}$ (h) Toluene

Figure 3 Solvent-induced inversion of excimer CPL. (a) A pyrene chromophore sandwiched between a pair of axially chiral binaphthyls forms twiststacked excimers of opposite chirality in polar and non-polar solvents. (b) Difference between left and right circularly polarized PL intensities $\left(\Delta l=I_{L}-I_{R}\right)$, and (c) glum values in different solvents show the inversion effect. Adapted with permission from Ref. 30. Copyright 2020 American Chemical Society.

$690 \mathrm{~nm}$. Interestingly, the dissymmetry factor showed a 2fold increase in methanol.

There have also been attempts to covalently attach multiple chromophores to a chiral scaffold to obtain CPL. Takaishi and co-workers used a quarternaphthyl skeleton on which naphthalene rings were arranged in way to define a helical axis. ${ }^{26 a}$ By functionalizing the naphthalene rings with an optimal number of pyrene residues, they were able to create conformationally rigid structures that supported a well-defined helical organization of chromophores (Figure 2a). These molecules displayed intense excimer CPL in both solution and solid state. Subsequently, the same group extended this approach to organize other fluorophores such as perylene and anthracene to create a library of CPL dyes spanning the visible spectrum. ${ }^{26 \mathrm{~b}}$ With the aid of time-dependent density functional theory calculations, the authors were able to establish a correlation between the sign of the CPL and the twist (chirality) in the excited-state structures. The above approach, though elegant, is synthetically demanding. A much simpler and perhaps a more general strategy was developed recently by Li et al. ${ }^{27}$ An achiral amphiphilic diblock copolymer was made to assume a helical structure in presence of a chiral additive. The helical polymeric structure was subsequently employed as the template for the organization of a variety of achiral fluorophores (Figure $2 \mathrm{~d}$ ). The polymer-fluorophore co-assembly exhibited an excimer CPL with the highest known dissymmetry value in the solid state, $g_{\text {lum }}=2.3 \times 10^{-2}$. Interestingly, the group also reported an antihelical effect, in which a right-handed polymer helix induced a left-handed CPL, and vice versa.

An interesting odd-even effect on the handedness of excimer CPL was reported in a series of chiral oligopeptides decorated with a pair of pyrene units. ${ }^{28}$ Pyrene units separated by an even number of intervening atoms gave rise to a negative CPL signal, and conversely for an odd number of atoms, the CPL signal turned positive in water. The depen- dence of the handedness of CPL emission on the arrangement of chromophores in the excimeric state opens up an avenue for the development of stimuli-responsive CPL dyes. This was first reported by Mimura et al. in a study on oligopeptides with a pair of pyrene pendant groups. ${ }^{29}$ The oligopeptides exhibited solvent-dependent sign inversion of CPL, even when the CD spectra showed no such change, thus highlighting a key difference between ground- and excitedstate solvation effects. More recently, a similar behaviour was also reported for an axially chiral molecule containing a pyrene moiety flanked between a pair of chiral binaphthyls (Figure 3). ${ }^{30}$ Hydroxyl substitution on binaphthyl rings had a marked influence on solvent-dependent intermolecular hydrogen bonding in the excited state. Consequently, the molecule showed a pronounced inversion of excimer chirality, from $g_{\text {lum }}$ of -0.012 in toluene to +0.012 in DMSO.

\section{Excimers in Light Harvesting}

In the context of light harvesting, long-lived excitons are highly desirable, because of their ability to transfer excitation energy over large distances. ${ }^{31}$ However in a multichromophoric system, long-lived excitons are also vulnerable to other relaxation processes that can occur on timescales faster than its lifetime. Self-trapping of Frenkel excitons into excimers is one such process that plagues exciton migration in multichromophoric systems. This is particularly true for perylene bisimide (PBI), ${ }^{32 a, b}$ an exceptional molecular dye with a very high molar extinction coefficient, an impressive chemical and photochemical stability, and an inherent tendency to form cofacially stacked H-aggregates. ${ }^{32 c}$ One of the earlier attempts to explain the self-trapping mechanism in PBI H-aggregates was undertaken by Engels and coworkers. ${ }^{33}$ They calculated the potential energy surfaces of ground and excited states of cofacially stacked PBI dimers 
(a)

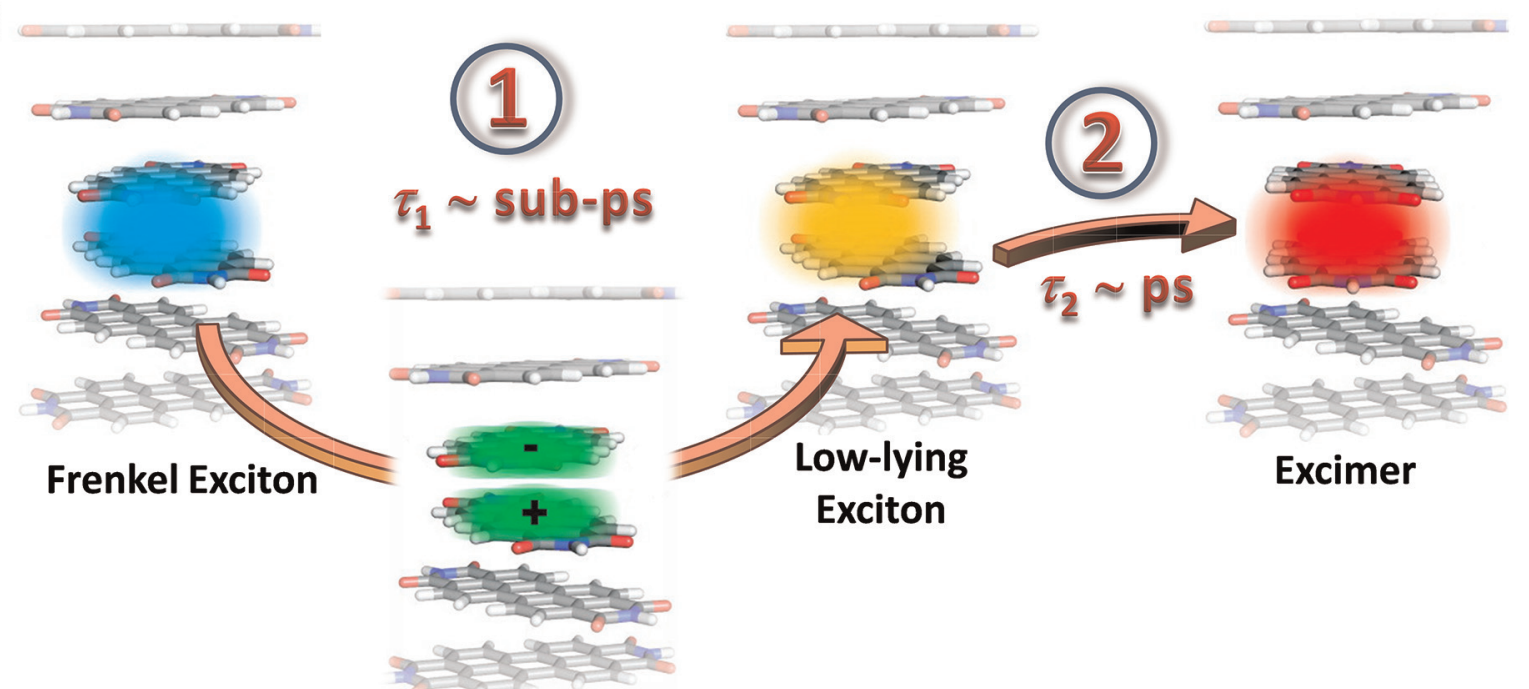

(b)

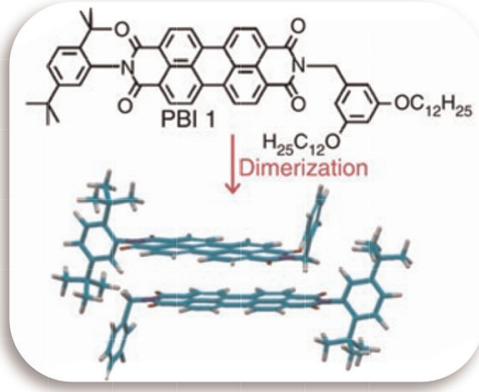

(e)

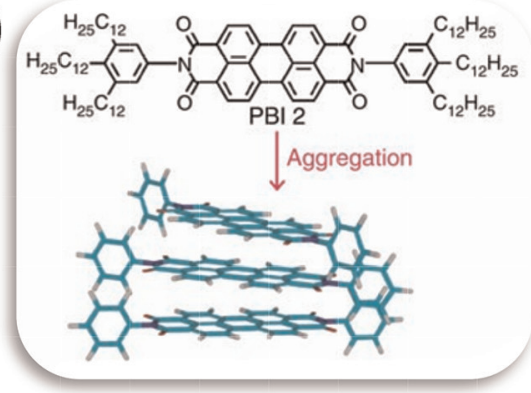

\section{Transient CT state}

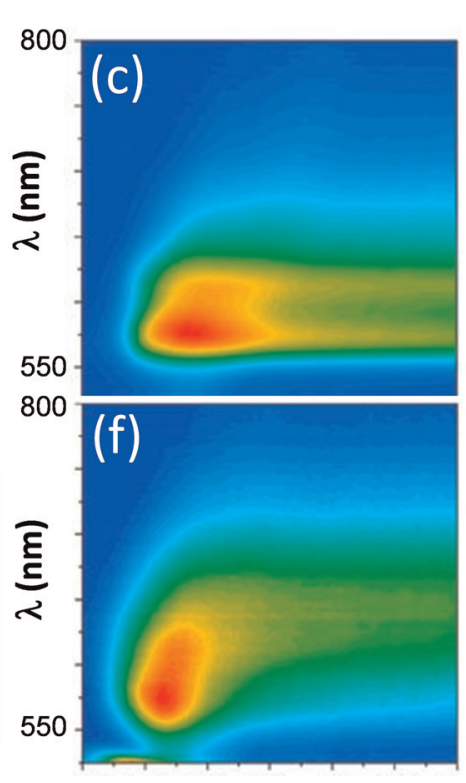

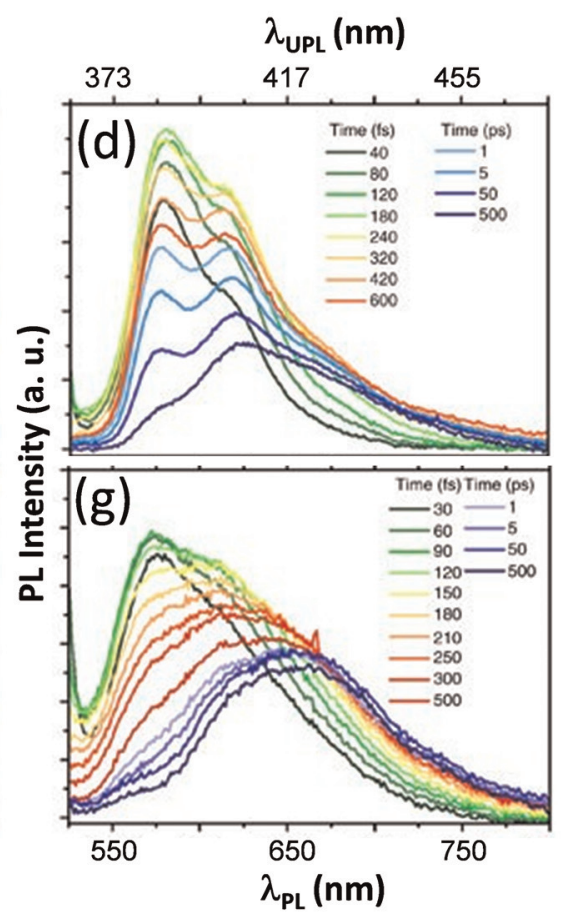

Figure 4 Excimer formation in PBI H-aggregates. a) Frenkel exciton rapidly (sub-picosecond) transitions into a lower energy exciton state through a transiently populated CT state. This is followed by a much slower (picoseconds) geometric rearrangement that reduces the twist angle. Adapted with permission from Ref. 34a. Copyright 2013 American Chemical Society. Femtosecond upconversion PL spectroscopy of dimeric (b, c, d) and extended (e, f, g) PBI H-aggregates reveals vibronically resolved PL from the Frenkel exciton in early stages (within $200 \mathrm{fs}$ ) after photoexcitation. Adapted from Ref. 41a published under a creative commons license (CC BY).

as a function of different structural parameters, such as the interplanar distance, longitudinal and transverse shifts, and the twist angle. Their calculations showed that the excimer formation process in PBI involved a considerable lowering of the twist angle between the stacked chromophores.
Though the authors stopped short of calling the self-trapped state as an excimer, in a subsequent work they modeled the process leading up to the geometry distortion in the excited state using time-dependent wavepacket calculations (Figure 4a). ${ }^{34}$ According to this model, the initial delocalized exciton 
undergoes an ultrafast localization onto a dimeric unit, which is followed by a crucial energy dissipation step. This stabilization of the Frenkel exciton is mediated through a transient gateway state with large CT character. Using femtosecond transient absorption (fsTA) spectroscopy, the timescale of this process was estimated to be of the order of $200 \mathrm{fs}$. Formation of a lower energy Frenkel exciton is followed by a much slower (picosecond) relaxation along the interstack torsional coordinate, which concludes the selftrapping process. The importance of configurational mixing between Frenkel and CT states was also proposed by Gao et al. to explain the broad, red-shifted excimer-like PL from PBI oligomers. ${ }^{35}$ The excited-state relaxation effects that aid the formation of excimers are not exclusively intermolecular in nature. In a recent work, Engels and co-workers investigated intra-monomer distortions associated with excimer formation, and its dependence on the extent of exciton delocalization. ${ }^{36}$

One of the more successful approaches to investigate the excimer formation process has been based on ultrafast spectroscopy on structurally rigid, covalently bridged dimers. ${ }^{37} \mathrm{~A}$ precise arrangement of chromophores in such model dimeric systems allowed one to systematically investigate the role of different structural aspects. Wasielewski and coworkers investigated the formation kinetics of excimers in a series of rigid, covalently bridged PBI dimers using fsTA spectroscopy. Their results established a direct correlation between excimer formation timescales and the strength of interchromophoric coupling. ${ }^{38}$ This work also presented an unambiguous way to probe the PBI excimer by monitoring its transition to higher lying CT states in the NIR, a spectral region that is usually free of interference from other excited-state absorption processes. Kim, Würthner and coworkers took the challenge further, and presented an interesting comparison of exciton dynamics and excimer formation in model PBI dimers vis-à-vis extended helical H-aggregates. With the help of transient absorption anisotropy decay measurements, they found excimer formation in large aggregates to be considerably slower $(40-50 \mathrm{ps})$ than that in dimers $(\sim 20 \mathrm{ps})$. A longer formation time was also found consistent with a distinctly slower rise $(\sim 300 \mathrm{ps})$ in the time-resolved PL. These results presented a compelling proof in favour of multimeric excimers in extended dye assemblies. ${ }^{39}$ The same group further quantified the effect of excimers on exciton mobility using exciton-exciton annihilation experiments. Their studies showed that Frenkel excitons migrate incoherently along an aggregated stack before getting trapped into the excimer state. The diffusion length was estimated to be in the range of 3-5 PBI units. ${ }^{40}$ Sung et al. employed femtosecond broadband fluorescence upconversion spectroscopy to investigate Frenkel exciton dynamics in helical PBI aggregates. ${ }^{41 a}$ An unprecedented temporal resolution allowed them to observe for the first time a vibronically resolved PL spectrum from the short-lived Frenkel exciton state (Figure $4 \mathrm{~b}-\mathrm{g})$. From the vibronic peak ratio $\left(I_{0-0} / I_{0-1}\right)$, it was estimated that the nascent exciton is coherently delocalized over at least 3 PBI units. ${ }^{41}$ A subsequent decrease in exciton coherence over a few hundred femtoseconds indicated the excimer formation process in PBI aggregates to be much faster than previously thought. This ultrafast selftrapping was attributed to an efficient mixing between Frenkel and CT exciton states. The detrimental effect of excimers is not solely limited to a reduced exciton mobility. Using model donor-acceptor foldameric systems, Fimmel et al. showed that excimers also impede photoinduced electron transfer across intramolecular $\mathrm{p} / \mathrm{n}$ heterojunctions. ${ }^{42}$

The studies reviewed so far stressed on the need to prevent excimer traps in order for $\mathrm{H}$-aggregates to support a long-range exciton transfer. A large family of PBI-based $\mathrm{H}-$ aggregates, where excimer formation is notoriously unavoidable, overwhelmingly supports this view. In this context, a notable exception was reported by Chaudhuri et al. $\mathrm{H}$-aggregated nanowires of cyclohexyl-appended PBI used in their study were uncharacteristically free of excimers. ${ }^{43}$ The low-temperature PL spectrum featured a pronounced vibronic progression, and a lifetime of 46 ns that is consistent with the long-lived Frenkel excitons of an H-type aggregate. While the factors contributing to this unprecedented stability of long-lived Frenkel excitons were not investigated, its implication in exciton migration was immediately clear. Local quenching sites (diameter $\sim 15 \mathrm{~nm}$ ) created on the nanowire surface using an Atomic force microscopy (AFM) tip could quench excitonic PL from nearly a micronsized area (Figure 5a). This indicated the ability of excitons to migrate over hundreds of nanometers, with an estimated exciton diffusion constant of $1.4 \times 10^{-2} \mathrm{~cm}^{2} / \mathrm{s}$ that is nearly 1-2 orders of magnitude higher than that for conjugated polymers. The possibility of suppressing excimer formation was also demonstrated by our group in extended H-aggregates of a PBI folda-dimer. ${ }^{44}$ The dimer which can exist in two distinct conformations: a non-interacting open and an intramolecularly stacked folded form, displayed a competition between two self-assembly pathways in solution. The faster pathway led to aggregates of the folded dimer with pronounced excimeric PL. These reorganize to form the more-stable excimer-free aggregates of the open conformer. A detailed investigation into possible factors that can prevent excimer formation in PBI H-aggregates was recently carried out by our group. We reported ambient-stable, bright, long-lived excitonic PL from an H-aggregated PBI, in both carefully grown single crystals and a relatively disordered solution self-assemblies. ${ }^{45}$ From the polarization dependence of absorption and PL spectra of single microcrystals, and correlating the results with the crystal structure, it was possible to identify two major factors that contributed to the stability of long-lived excitons against excimer formation. An unusually large exciton splitting $\left(\sim 1265 \mathrm{~cm}^{-1}\right)$, resulting from the combination of H-type Coulombic and J- 


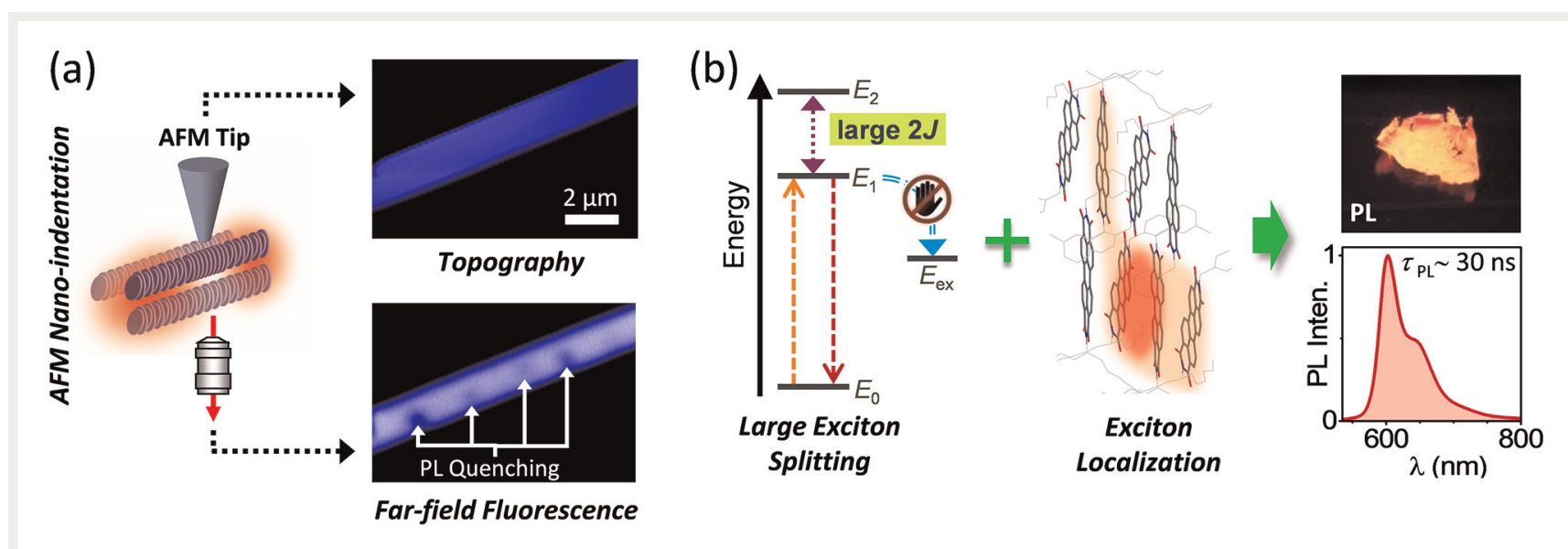

Figure 5 Escaping excimeric traps in PBI H-aggregates. a) Correlated AFM topography and far-field PL microscopy suggest nearly micron-scale exciton migration in excimer-free H-aggregates of PBI. Adapted with permission from Ref. 43. Copyright 2011 American Chemical Society. b) A combination of very large exciton splitting (2J) and an efficient exciton localization safeguards Frenkel excitons from excimer traps, resulting in a bright, vibronically resolved PL. Adapted from Ref. 45 published under a creative commons license (CC BY-NC).

type CT interaction ( $\mathrm{Hj}$ coupling), stabilized the lowest-energy Frenkel exciton, thus inhibiting its crossover to the excimer state (Figure 5b). A recent theoretical work on covalent PBI dimers also suggested an upper threshold of CT contribution $(\sim 58 \%)$, above which excimer formation in cofacial PBI aggregates could be suppressed. ${ }^{46}$ In addition to the large exciton splitting, the exciton wavefunction also experiences an efficient self-localization that further safeguards it from potential trap sites in the vicinity. In view of these findings, it is prudent to take special note of two recent studies. A very large exciton splitting of $1230 \mathrm{~cm}^{-1}$ was also reported for an H-aggregated PBI crystal by Austin et al. ${ }^{47}$ Though PL characteristics of the crystals were not investigated, these crystals could make an interesting system to study the influence of large exciton splitting on the fate of excimers. In another work, Würthner's group reported a supramolecular polymorph of a PBI dye that featured a broad absorption spectrum and a vibronically resolved steady-state PL spectrum. ${ }^{48}$ Both these systems and the one we studied shared certain similarities in molecular packing: a twisted stacking of PBI units with a longitudinal shift. Such chromophoric arrangement can promote a strong coupling between Coulombic and CT interactions, ${ }^{49}$ which can be crucial towards stabilizing the exciton against excimeric trapping.

Notwithstanding the undesirable influence of excimers on exciton migration, there also exists an alternate perspective that highlights the positive role of excimers in singlet fission (SF). SF refers to a spin-allowed process in which a photoexcited singlet exciton interacts with a neighboring molecule in the ground state to generate two lower energy triplet excitons. ${ }^{50}$ In the context of photovoltaics, generating multiple excitons (electron-hole pairs) through SF can push the efficiency of a solar cell well above the Shockley-Queiss- er limit of $\sim 33 \%$. SF proceeds through an excited state that is best described as a coherent superposition of excitoncoupled singlet excited $\left.\left.\right|^{1}\left(S_{1} S_{0}\right)\right\rangle$, charge-transfer $|\mathrm{CT}\rangle$ and correlated triplet pair $\left|{ }^{1}\left(T_{1} T_{1}\right)\right\rangle$ states. ${ }^{37 e}$ With two out of these three interactions also operating in excimers, it is only natural that excimers could influence the fate of SF.

The role of a short-lived excimer intermediate was first proposed theoretically by Zimmerman et al. to explain SF in pentacene. ${ }^{51}$ An interaction between excited- and groundstate pentacene molecules was proposed to facilitate a state crossing from the molecular singlet excited state to a dimeric multiexcitonic dark state, with the characteristics of a correlated triplet pair. This key step is believed to be mediated through a transient excimer-like species that holds the two molecular units together. Once the multiexcitonic state is formed, the repulsive interactions lead to the separation of two pentacene monomers, each with a triplet exciton localized on it. Friend and coworkers experimentally confirmed excimer-mediated SF in concentrated solution of TIPS-pentacene molecules, ${ }^{52}$ which showed a near-unity quantum yield: two triplet excitons for every absorbed photon. By comparing the rate of growth of triplet states vis-à-vis the decay of excimer PL, they proposed a two-step phenomenological model for SF: a fast, diffusion-limited formation of excimers, followed by its dissociation to generate two triplet excitons on a timescale of $400-530$ ps. The importance of CT interactions in SF involving donor-acceptor chromophores was investigated in a series of thiophene-diketopyrrolopyrrole-based molecular crystals. ${ }^{53}$ Here too, a robust correlation between intermolecular donor-acceptor interactions, excimer decay rates and SF efficiencies confirmed the involvement of an intermediate excimer state with a large CT character (Figure 6a). The intermediary role 
(a) Mediated by Excimers

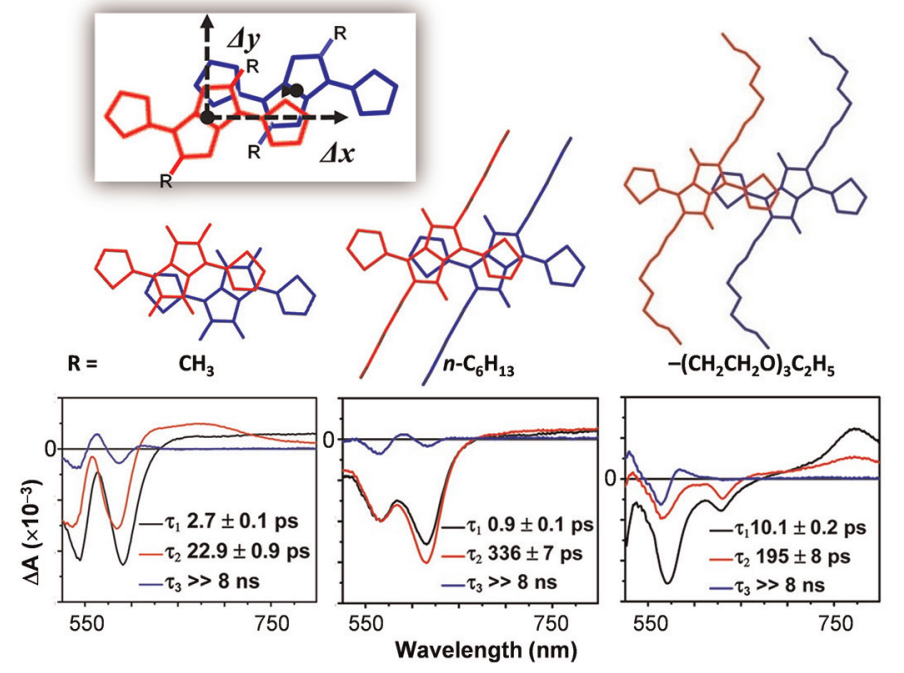

(b) Independent of Excimers
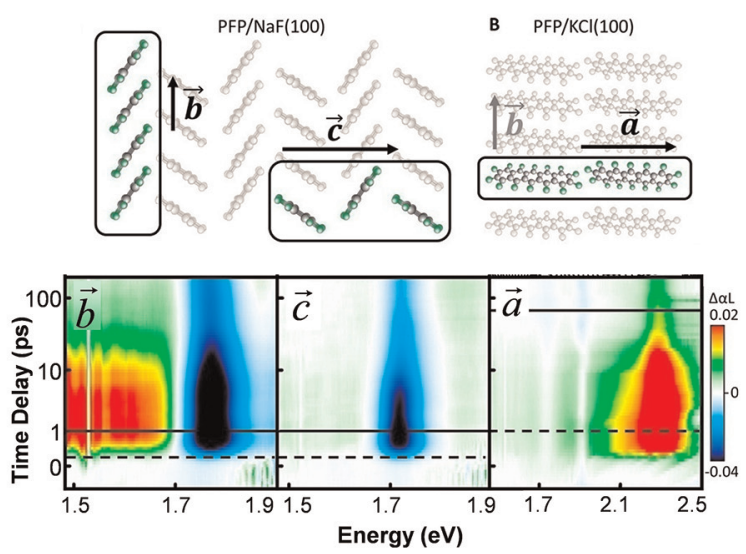

(c) Hindered by Excimers
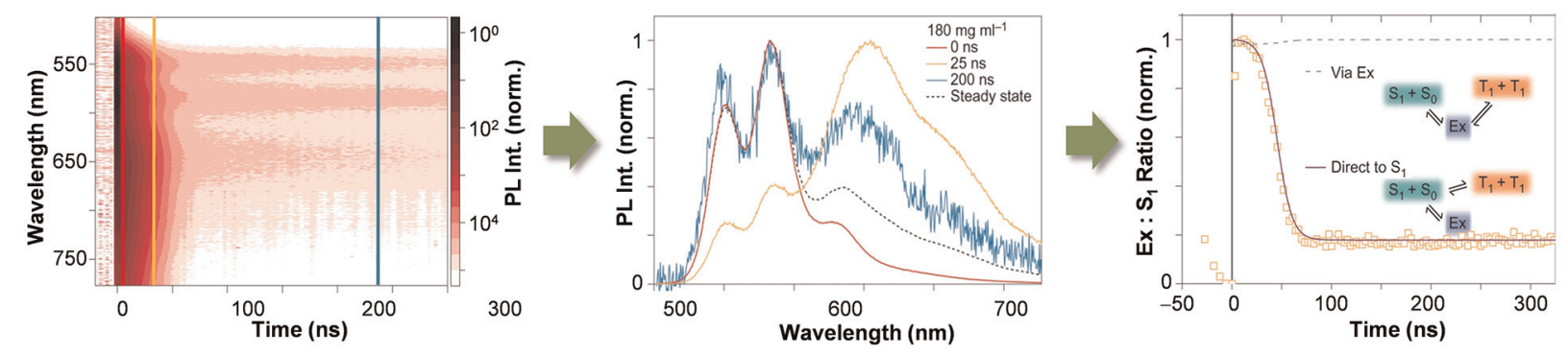

Figure 6 Excimers in SF. a) Excimer-mediated exothermic SF in polycrystalline thin films of diketopyrrolopyrrole derivatives with slip-stacked packing. Globally fit species-associated spectra show strongly correlated excimer $\left(\mathrm{T}_{1}\right)$ and triplet formation $\left(\mathrm{T}_{2}\right)$ processes. Adapted with permission from Ref. 53 . Copyright 2016 American Chemical Society. b) SF in perfluoropentacene single-crystals happens exclusively along the $b$-axis. False color plots showing evolution of various differential absorption features, corresponding to disappearance of Frenkel exciton (1.7-1.8 eV), appearances of excimer (1.5$1.7 \mathrm{eV})$ and correlated triplet pair (2.1-2.5 eV). Kinetic analysis shows excimers neither compete nor assist SF. Adapted with permission from Ref. 54. Copyright 2014 American Chemical Society. c) Time-resolved PL of a tetracene derivative shows appearance of excimers at intermediate timescales ( $25 \mathrm{~ns}$ ). Decay profile of excimers to singlet excitons suggests a competition between excimer formation and SF processes. Adapted with permission from Ref. 58. Copyright 2018 Macmillan Publishers Limited, part of Springer Nature.

of excimers in SF is however not universally true. A notable exception was reported by Kolata et. al., who observed contrasting SF efficiencies along two different crystallographic axes of perfluoropentacene single crystals. ${ }^{54}$ SF happened exclusively along the $b$-axis, where molecules arranged in a slip-stacked (J-type) fashion, but was suppressed along the $a$-axis that supported a face-to-edge (herringbone) packing. Interestingly, fsTA spectroscopy results showed that the correlated triplet pair, $\left.\left.\right|^{1}\left(T_{1} T_{1}\right)\right\rangle$, is formed directly from the exciton-coupled singlet $\left.\left.\right|^{1}\left(S_{1} S_{0}\right)\right\rangle$ state, through a process that precedes excimer formation by $\sim 300 \mathrm{fs}$ (Figure $6 \mathrm{~b}$ ). This clearly showed that excimers in perfluoropentacene crystals neither compete with nor mediate in SF.

The examples discussed so far were limited to materials that exhibit an exothermic SF, satisfying the energy criterion of $E\left(S_{1}\right)>2 E\left(T_{1}\right)$. While exothermic SF can show very high triplet yields, the energy of the resultant triplet state is often too low to be practically useful. In this context, endothermic SF offers a useful alternative with higher energy conversion efficiencies. The role of excimers in endothermic SF remains intensely debated, with more results suggesting their detrimental influence. Friend and co-workers used TA and timeresolved PL to monitor the process in concentrated solution of TIPS-tetracene, a chromophore in which $S_{1}$ and $T_{1}$ energies satisfy the criterion for endothermic SF. ${ }^{55}$ An ultrafast conversion of the photoexcited singlet state into an excimer state was observed on a timescale of $<100$ ps. Interestingly, the excimer displayed a triplet absorption and a singlet emission, which is consistent with the characteristic of a bound pair of correlated triplets. The excimer would eventu- 
ally dissociate generating a pair of separated triplet excitons. A contrasting picture emerged from the work of Korovina et al., who investigated SF in a pair of covalently bridged tetracene dimers with a varying degree of interchromophoric interaction. ${ }^{56}$ In the strongly coupled dimer, the excimer state formed rapidly ( $\sim 180 \mathrm{fs}$ ) that promptly decayed to the ground state without forming triplet excitons. The weakly coupled dimer, on the other hand, showed an efficient conversion of the singlet exciton to a correlated triplet pair, through a process that completely bypassed the excimer intermediate. That excimers could be detrimental to SF also found support in theoretical studies. ${ }^{57}$ Dover et al. carried out solution-phase investigations of endothermic SF and its exact reverse process, that is triplet-triplet annihilation (TTA) for a tetracene derivative. ${ }^{58}$ The upconverted PL spectrum generated by sensitized TTA did not show any appreciable excimer signature. Further, by fitting the time-dependence of molecular $\left(S_{1}\right)$ to excimer PL intensities to different kinetic models (Figure 6c), it was found that excimers were 14 times more likely to decay to the ground state than undergo SF. A recent study by Kim and co-workers on covalent PBI dimers, also an endothermic SF material, ${ }^{59}$ further highlighted the deleterious effect of excimers in multiexciton generation, a key step in the SF process. ${ }^{60}$ All three dimers used in the study supported a strong H-type Coulombic and varying degrees of J-type CT coupling. A weaker CT coupling was found to encourage the formation of an excimer trap ( $200 \mathrm{fs})$ that inhibited its further transition to the multiexcitonic state. Conversely, dimers with strong CT coupling readily relaxed into the multiexcitonic state, completely bypassing the excimer. The rate of CT-mediated transition to the multiexcitonic state further showed an order of magnitude increase in polar solvents, thus highlighting the importance of CT interactions. A similar CT-mediated transfer between singlet and correlated triplet pair states was also reported in slip-stacked dimers of terrylenediimide (TDI), which is incidentally an exothermic SF material. ${ }^{61 a}$ Here too, excimer formation was shown to inhibit SF. A competition between CT-mediated SF and excimer formation was also studied by the same group in different polycrystalline thin films of TDI. ${ }^{61 \mathrm{~b}}$ They investigated the role of molecular packing to show that a twisted stacking between TDI chromophores led to $190 \%$ triplet yield, while a slip-stacked packing predominantly favoured excimer formation. The instances discussed above allow one to draw a few conclusions about the contrasting role of excimers in SF. Excimer-mediated SF requires a strong coupling between the excimer and the correlated triplet state. Thus, a fast structural relaxation that stabilizes the excimer state and lowers its energy significantly is likely to weaken its coupling with the correlated triplet state, and negatively impact the SF process. The problem is expected to be more severe in case of endothermic SF, where the energy of the correlated triplet state is comparatively higher than that of the excimer.
Power conversion efficiency of a photovoltaic cell is directly proportional to its open circuit voltage, a quantity that is usually very low for most organic photovoltaic systems. Bartynski et al. showed that symmetry-breaking charge separation (SBCS) between closely interacting pair of identical chromophores can significantly increase the open circuit voltage, and lower recombination losses. ${ }^{62}$ SBCS is a process by which one excited chromophore interacts with the other in ground state to create a charge-separated radical ion-pair, wherein the electron and the hole separately reside on two different molecules. ${ }^{63}$ SBCS also plays a key role in certain natural photosynthetic reaction centers. ${ }^{64}$ Since the process requires an interaction between excited- and ground-state molecules, the possibility of excimers interfering with SBCS looms large. In polycyclic aromatic chromophores, excimers typically involve molecular stacking, which makes it possible to dissuade excimer formation and promotes SBCS by bringing multiple chromophores in a closely interacting, but a non-stacked, arrangement. This was successfully demonstrated in a couple of cyclic PBI dimers and trimers, where chromophores were either widely separated using appropriate spacers, or arranged in a rigid triangular geometry. In both instances, it was possible to discourage structural relaxation that could lead to deep excimer traps, resulting in SBCS with a high quantum yield. ${ }^{65}$ A notable contribution was made by Würthner and coworkers, who attempted to distinguish between "shallow" excimer-like states and structurally relaxed deep excimer traps in the context of SBCS. The PBI cyclophane used in their study exhibited interesting solvent-dependent behaviour (Figure 7a). In $\mathrm{CHCl}_{3}$, a strong correlation was observed between the rise time of $\mathrm{PBI}^{\circ-}$ radical anion and the decay of excimer PL, suggesting that the excimer could be an intermediary in SBCS. ${ }^{66}$ In THF however, a tighter stacking between the two PBI units resulted in the formation of a structurally relaxed deep excimer state that fully suppressed SBCS. A recent report on polycrystalline thin films of $\mathrm{PBI}$ presented a new perspective on the role of interchromophoric coupling in excimer formation. ${ }^{67}$ Subtle differences in the slip-stacked packing pattern manifested itself in varying degrees of Coulombic and CT coupling, which in turn modulated the CT rate between PBI chromophores. Consequently, SBCS was favoured over excimer formation in films that supported a stronger CT interaction (Figure 7b). The competition between excitoncoupled and charge-separated states can also be tilted in favour of the latter in more polar solvents, as was shown using different model bichromophoric systems. ${ }^{68}$ More recently, Kim and co-workers reported that in highly polar solvents, the CT resonance character of deep excimer trap of a baysubstituted PBI cyclophane could be sufficiently enhanced to achieve an incomplete SBCS. ${ }^{69}$

Finally, in the context of solar energy harvesting, underutilization of the low-energy part of the solar spectrum has been an outstanding concern. TTA-based photon upconver- 
(a)
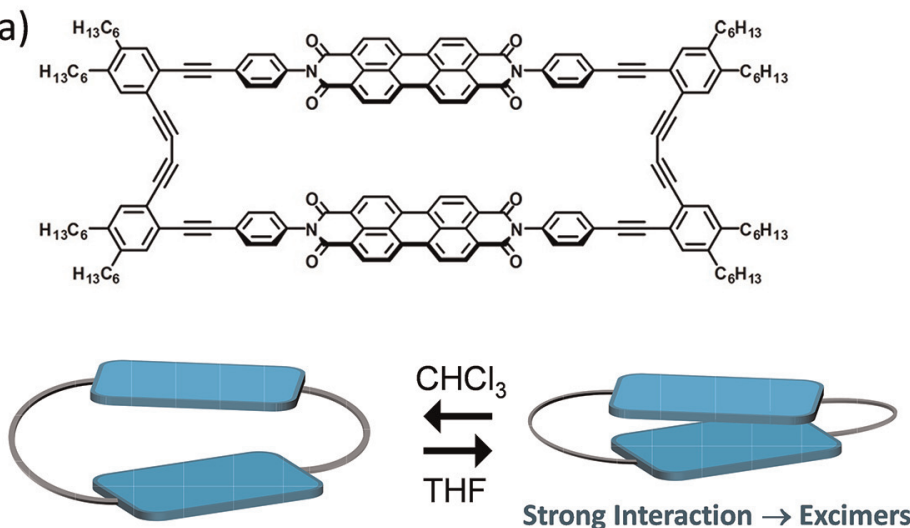

Weak Interaction $\rightarrow$ SBCS

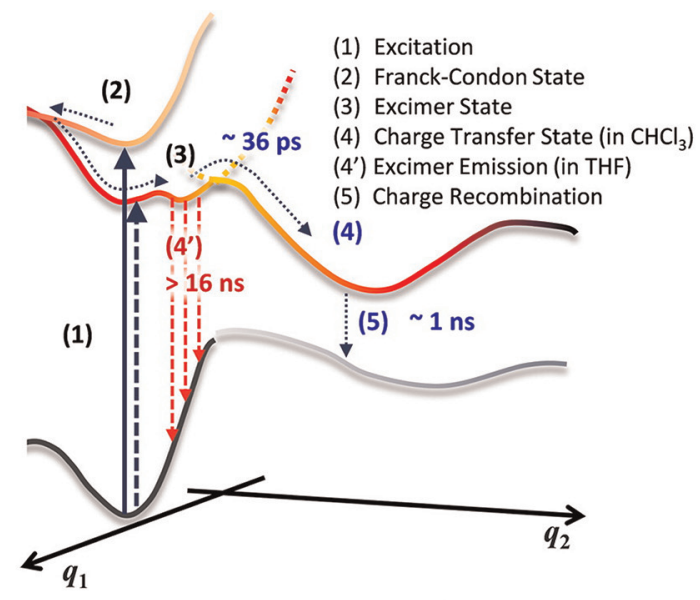

(b)

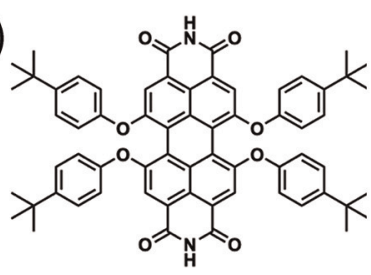
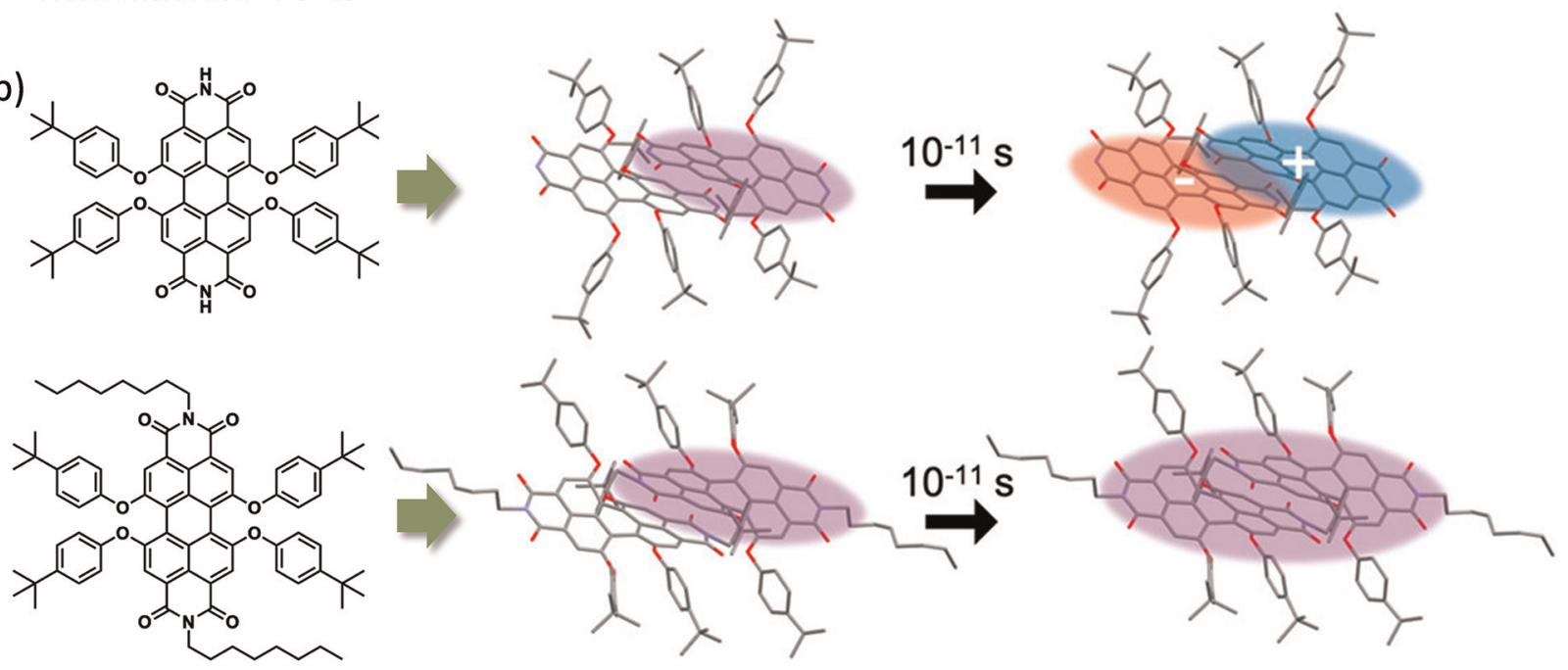

Figure 7 Competition between excimer formation and SBCS. a) The competition between formation of deep excimer traps and SBCS in PBI cyclophane is dependent on the strength of interchromophoric coupling. The schematic energy level diagram highlights the important processes and their respective timescales. Adapted with permission from Ref. 66. Copyright 2016 American Chemical Society. b) Nature of molecular packing in polycrystalline thin films of two bay-substituted PBI derivatives controls the interactions between PBI chromophores and the fate of the photoexcited state; imidehydrogenated derivative (top) supports SBCS, whereas strong mixing of singlet exciton and CT states in the di-octyl derivative (bottom) favours excimer formation. Adapted with permission from Ref. 67. Copyright 2020 American Chemical Society.

sion offers a potential solution to address this loss. ${ }^{70}$ Here too, formation of excimers competes against upconversion of triplet to singlet excitons, and contributes to a significant energy loss. Börjesson's group investigated sensitized TTA upconversion in a perylene dye, and elucidated the role of a triplet dimer $\left(T_{1} S_{0}\right)$ precursor in excimer formation. ${ }^{71} \mathrm{~A}$ significant increase in the upconversion yield was reported by suppressing excimer formation in less polar solvents.

\section{Conclusions and Outlook}

This review started with the aim to showcase excimers in a new light, one that is shaped by the recent developments in the area of organic multichromophoric systems. In the first four decades after its discovery, excimers were often viewed in isolation: as a transient excited-state species that resulted from, and therefore reported on, closely interacting chromophores. This outlook changed completely in the last decade, as the ability to probe the dynamics of excited states witnessed a marked improvement. This widened our perception of what excimers are, and how they might influence different photophysical and photochemical processes in multi- 
chromophoric systems. When viewed in the larger context of the electronic interactions that define it, the excimer appears to be an integral part of all such excited-state phenomena and processes that involve exciton coupling, charge transfer interactions and excited-state structural relaxations of chromophores. Nowhere is this connection more obvious than in the area of light harvesting, where we are only beginning to unravel the role of excimers, in processes such as exciton migration, SF and SBCS. In judging whether excimers are useful or detrimental, there is clearly a need to avoid generalizations. We have discussed systems where a strong mixing between exciton-coupled and CT states, combined with geometric relaxations, can lead to the formation of low-energy excimers. Such deep excimers can indeed trap mobile excitons, and inhibit SF and SBCS. In contrast, a shallow excimer state is often suitably poised to act as a key intermediate in the same processes. Therefore, going forward, an ability to fine tune the characteristics of the excimer state through molecular design appears to be a goal worth pursuing. Structurally well-defined dimers have served as useful model systems in the systematic investigation of factors controlling different aspects of excimer formation. However, there is a need to extend that approach to large, supramolecular dye aggregates. An obvious challenge lies in the form of large-scale structural heterogeneities and lack of long-range order in these aggregates. To this end, investigation of welldefined oligomeric systems and crystalline aggregates can play an important role. Recently, Kim and co-workers studied the structural evolution during SBCS in a PBI-based donor-acceptor-donor molecule, using time-resolved Raman spectroscopy. ${ }^{72}$ This unique perspective into the structural changes associated with excited-state processes will pave way for a better understanding of structure-property relationships in complex multichromophoric systems.

Finally, the examples that we have reviewed do not explicitly account for temporal fluctuations in interchromophoric interactions, which might result from molecular dynamics on the timescale of seconds. It is well known that an exciton in extended multichromophoric systems is not a static unit. Following every absorption event, the exciton can randomly localize on different segments of the larger multichromophoric system. ${ }^{73}$ This can introduce large-scale fluctuations in the nature of exciton coupling and CT interactions that define an excimer. Recently, Lupton and coworkers confirmed such temporal fluctuations in excimeric interactions using single-molecule PL spectroscopy on rigid bichromophoric systems. ${ }^{74}$ To what extent such fluctuations may influence processes such as SF and SBCS is a question that could stimulate future quests.

\section{Acknowledgements}

D. C. acknowledges Lakshmi Narsimhan for the MATLAB program file (https://www.mathworks.com/matlabcentral/fileexchange/27358-ripple-formation-on-the-surface-of-water), which was modified and used to create the background for the graphic abstract.

\section{Conflict of Interest}

The author declares no conflict of interest.

\section{References}

(1) Szent-Györgyi, A. Science 1941, 93, 609.

(2) (a) McConnell, H. M.; Hoffman, B. M.; Metzger, R. M. Proc. Natl. Acad. Sci. U.S.A. 1965, 53, 46. (b) Silbey, R. Annu. Rev. Phys. Chem. 1976, 27, 203.

(3) (a) Kenorkian, J.; Labes, M. M.; Larson, D. C.; Wu, D. C. Spec. Discuss. Faraday Soc. 1971, 51, 139. (b) Dodablapur, A.; Torsi, L.; Katz, H. E. Science 1995, 268, 270.

(4) (a) Heeger, A. J.; Kivelson, S.; Schrieffer, J. F.; Su, W. P. Rev. Mod. Phys. 1988, 60, 781. (b) Collini, E.; Scholes, G. D. Science 2009, $323,369$.

(5) (a) De Schryver, F. C.; Vosch, T.; Cotlet, M.; Van der Auweraer, M.; Müllen, K.; Hofkens, J. Acc. Chem. Res. 2005, 38, 514. (b) Serin, J. M.; Brousmiche, D. W.; Fréchet, J. M. J. Chem. Commun. 2002, 2605.

(6) (a) Tamiaki, H.; Miyatake, T.; Tanikaga, R.; Holzwarth, A. R.; Schaffner, K. Angew. Chem. Int. Ed. 1996, 35, 772. (b) Zang, L.; Che, Y.; Moore, J. Acc. Chem. Res. 2008, 41, 1596. (c) Wasielewski, M. R. Acc. Chem. Res. 2009, 42, 1910.

(7) Spano, F. C. Annu. Rev. Phys. Chem. 2006, 57, 217.

(8) Friend, R. H.; Gymer, R. W.; Holmes, A. B.; Burroughes, J. H.; Marks, R. N.; Taliani, C.; Bradley, D. D. C.; Dos Santos, D. A.; Brédas, J. L.; Lögdlund, M.; Salaneck, W. R. Nature 1999, 397, 121.

(9) Coakley, K. M.; McGehee, M. D. Chem. Mater. 2004, 16, 4533.

(10) (a) Clark, J.; Silva, C.; Friend, R. H.; Spano, F. C. Phys. Rev. Lett. 2007, 98, 206406. (b) Cornil, J.; Beljonne, D.; Calbert, J. P.; Brédas, J.-L. Adv. Mater. 2001, 13, 1053.

(11) (a) Matsui, A. H. Pure Appl. Chem. 1995, 67, 429. (b) Cornil, J.; Beljonne, D.; Coropceanu, V.; Brédas, J.-L. Chem. Rev. 2004, 104, 4971. (c) Spano, F. C. Acc. Chem. Res. 2010, 43, 429.

(12) (a) Grover, M.; Silbey, R. J. Chem. Phys. 1971, 54, 4843. (b) Hennebicq, E.; Pourtois, G.; Scholes, G. D.; Herz, L. M.; Russell, D. M.; Silva, C.; Setayesh, S.; Grimsdale, A. C.; Müllen, K.; Brédas, J.-L.; Beljonne, D. J. Am. Chem. Soc. 2005, 127, 4744. (c) Brédas, J.-L.; Sargent, E. H.; Scholes, G. D. Nat. Mater. 2017, 16, 35.

(13) Birks, J. B. Rep. Prog. Phys. 1975, 38, 903.

(14) Förster, T.; Kaspar, K. Z. Phys. Chem. 1954, 1, 275.

(15) (a) Saigusa, H.; Sun, S.; Lim, E. C. J. Phys. Chem. 1992, 96, 2083. (b) Saigusa, H.; Lim, E. C. Acc. Chem. Res. 1996, 29, 171.

(16) (a) Jenekhe, S. A.; Osaheni, J. A. Science 1994, 265, 765. (b) Conwell, E. Trends Polym. Sci. 1997, 7, 218. (c) Schwartz, B. J. Annu. Rev. Phys. Chem. 2003, 54, 141.

(17) (a) Förster, T. Pure Appl. Chem. 1962, 4, 121. (b) Förster, T. Pure Appl. Chem. 1963, 7, 73. (c) Murrell, J. N.; Tanaka, J. Mol. Phys. 1964, 7, 363. (d) Azumi, T.; Armstrong, A. T.; McGlynn, S. P. 
J. Chem. Phys. 1964, 41, 3131. (e) Azumi, T.; McGlynn, S. P. J. Chem. Phys. 1964, 41, 3839.

(18) (a) Stevens, B.; Ban, M. I. J. Chem. Soc. 1964, 60, 1515. (b) Birks, J. B. J. Phys. Chem. 1963, 67, 2199. (c) Birks, J. B.; Chistophorou, L. G. Nature 1962, 194, 442.

(19) Stevens, B.; Hutton, E. Nature 1960, 186, 1045.

(20) (a) Kim, Y.; Bouffard, J.; Kooi, S. E.; Swager, T. M. J. Am. Chem. Soc. 2005, 127, 13726. (b) Williams, E. L.; Haavisto, K.; Li, J.; Jabbour, G. E. Adv. Mater. 2007, 19, 197. (c) Liu, H.; Yao, L.; Li, B.; Chen, X.; Gao, Y.; Zhang, S.; Li, W.; Lu, P.; Yang, B.; Ma, Y. Chem. Commun. 2016, 52, 7356. (d) Luo, Q.; Li, L.; Ma, H.; Lv, C.; Ziang, X.; Gu, X.; An, Z.; Zou, B.; Zhang, C.; Zhang, Y. Chem. Sci. 2020, 11, 6020. (e) Chen, J.; Tang, N.; Zhou, J.; Wang, L.; Jiang, N.; Zheng, N.; Liu, L.; Xie, Z. J. Phys. Chem. Lett. 2021, 12, 3373.

(21) Teo, Y. N.; Kool, E. T. Chem. Rev. 2012, 112, 4221.

(22) (a) Kumar, J.; Nakashima, T.; Tsumatori, H.; Mori, M.; Naito, M.; Kawai, T. Chem. Eur. J. 2013, 19, 14090. (b) Kumar, J.; Nakashima, T.; Kawai, T. J. Phys. Chem. Lett. 2015, 6, 3445.

(23) Brittain, H.; Fendler, J. H. J. Am. Chem. Soc. 1980, 102, 6372.

(24) (a) Inouye, M.; Hayashi, K.; Yonenaga, Y.; Itou, T.; Fujimoto, K.; Uchida, T.; Iwamura, M.; Nozaki, K. Angew. Chem. Int. Ed. 2014, 53, 14392. (b) Hayashi, K.; Miyaoka, Y.; Ohishi, Y.; Uchida, T.; Iwamura, M.; Nozaki, K.; Inouye, M. Chem. Eur.J. 2018, 24, 14613.

(25) Miki, K.; Noda, T.; Gon, M.; Tanaka, K.; Chujo, Y.; Mizuhata, Y.; Tokitoh, N.; Ohe, K. Chem. Eur. J. 2019, 25, 9211.

(26) (a) Takaishi, K.; Takehana, R.; Ema, T. Chem. Commun. 2018, 54, 1449. (b) Takaishi, K.; Iwachido, K.; Takehana, R.; Uchiyama, M.; Ema, T. J. Am. Chem. Soc. 2019, 141, 6185.

(27) Li, Q.; Yuan, J.; Liang, H.; Zheng, F.; Lu, X.; Yu, C.; Lu, Q. ACS Nano 2020, 14, 8939.

(28) Mimura, Y.; Motomura, Y.; Kitamatsu, M.; Imai, Y. Tetrahedron Lett. 2020, 61, 152238.

(29) Mimura, Y.; Kitamura, S.; Shizuma, M.; Kitamatsu, M.; Fujiki, M.; Imai, Y. Chem Select 2017, 2, 7759.

(30) Takaishi, K.; Iwachido, K.; Ema, T. J. Am. Chem. Soc. 2020, 142, 1774.

(31) (a) Rocke, C.; Zimmermann, S.; Wixforth, A.; Kotthaus, J. P.; Bohm, G.; Weimann, G. Phys. Rev. Lett. 1997, 78, 4099. (b) Scully, S. R.; Armstrong, P. B.; Edder, C.; Frechet, J. M. J.; McGehee, M. D. Adv. Mater. 2007, 19, 2961. (c) Guillet, T.; Berrehar, J.; Grousson, R.; Kovensky, J.; L-Mayer, C.; Schott, M.; Voliotis, V. Phys. Rev. Lett. 2001, 87, 087401.

(32) (a) Marciniak, H.; Li, X.-Q.; Würthner, F.; Lochbrunner, S. J. Phys. Chem. A 2011, 115, 648. (b) Ramanan, C.; Kim, C. H.; Marks, T. J.; Wasielewski, M. R. J. Phys. Chem. C. 2014, 118, 16941. (c) Würthner, F.; Saha-Möller, C. R.; Fimmel, B.; Ogi, S.; Leowanawat, P.; Schmidt, D. Chem. Rev. 2016, 116, 962.

(33) Fink, R. F.; Seibt, J.; Engel, V.; Renz, M.; Kaupp, M.; Lochbrunner, S.; Zhao, H.-M.; Pfister, J.; Würthner, F.; Engels, B. J. Am. Chem. Soc. 2008, 130, 12858.

(34) (a) Schubert, A.; Settels, V.; Liu, W.; Würthner, F.; Meier, C.; Fink, R. F.; Schindlbeck, S.; Lochbrunner, S.; Engels, B.; Engel, V. J. Phys. Chem. Lett. 2013, 4, 792. (b) Settels, V.; Schubert, A.; Tafipolski, M.; Liu, W.; Stehr, V.; Topczak, A. K.; Pflaum, J.; Deibel, C.; Fink, R. F.; Engel, V.; Engels, B. J. Am. Chem. Soc. 2014, 136, 9327. (c) Bellinger, D.; Pflaum, J.; Brüning, C.; Engel, V.; Engels, B. Phys. Chem. Chem. Phys. 2017, 19, 2434.

(35) Gao, F.; Zhao, Y.; Liang, W. J. Phys. Chem. B 2011, 115, 2699.

(36) (a) Deutsch, M.; Wirsing, S.; Kaiser, D.; Fink, R. F.; Tegeder, P.; Engels, B. J. Chem. Phys. 2020, 153, 224104. (b) Diehl, F. P.; Roos, C.; Duymaz, A.; Lunkenheimer, B.; Köhn, A.; Basché, T. J. Phys. Chem. Lett. 2014, 5, 262.
(37) (a) De Schryver, F. C.; Collart, P.; Vandendriessche, J.; Goedeweeck, R.; Swinnen, A.; Van der Auweraer, M. Acc. Chem. Res. 1987, 20, 159. (b) Giaimo, J. M.; Lockard, J. V.; Sinks, L. E.; Scott, A. M.; Wilson, T. M.; Wasielewski, M. R. J. Phys. Chem. A 2008, 112, 2322. (c) Lindquist, R. J.; Lefler, K. M.; Brown, K. E.; Dyar, S. M.; Margulies, E. A.; Young, R. M.; Wasielewski, M. R. J. Am. Chem. Soc. 2014, 136, 14912. (d) Engels, B.; Engel, V. Phys. Chem. Chem. Phys. 2017, 19, 12604. (e) Young, R. M.; Wasielewski, M. R. Acc. Chem. Res. 2020, 53, 1957.

(38) (a) Lindquist, R. J.; Lefler, K. M.; Brown, K. E.; Dyar, S. M.; Margulies, E. A.; Young, R. M.; Wasielewski, M. R. J. Am. Chem. Soc. 2014, 136, 14912. (b) Brown, K. E.; Salamant, W. E.; Shoer, L. E.; Young, R. M.; Wasielewski, M. R. J. Phys. Chem. Lett. 2014, 5, 2588.

(39) Lim, J. M.; Kim, P.; Yoon, M.-C.; Sung, J.; Dehm, V.; Chen, Z.; Würthner, F.; Kim, D. Chem. Sci. 2013, 4, 388.

(40) Son, M.; Park, K. H.; Shao, C.; Würthner, F.; Kim, D. J. Phys. Chem. Lett. 2014, 5, 3601.

(41) (a) Sung, J.; Kim, P.; Fimmel, B.; Würthner, F.; Kim, D. Nat. Commun. 2015, 6, 8646. (b) Kaufmann, C.; Kim, W.; Nowak-Król, A.; Hong, Y.; Kim, D.; Würthner, F. J. Am. Chem. Soc. 2018, 140, 4253.

(42) (a) Fimmel, B.; Son, M.; Sung, Y. M.; Grüne, M.; Engels, B.; Kim, D.; Würthner, F. Chem. Eur. J. 2015, 21, 615. (b) Nowak-Krol, A.; Fimmel, B.; Son, M.; Kim, D.; Würthner, F. Faraday Discuss. 2015, $185,507$.

(43) Chaudhuri, D.; Li, D.; Che, Y.; Shafran, E.; Gerton, J. M.; Zang, L.; Lupton, J. M. Nano Lett. 2011, 11, 488.

(44) Samanta, S.; Chaudhuri, D. J. Phys. Chem. Lett. 2017, 8, 3427.

(45) Samanta, S.; Ray, S. K.; Deolka, S.; Saha, S.; Pradeep, K. R.; Bhowal, R.; Ghosh, N.; Chaudhuri, D. Chem. Sci. 2020, 11, 5710.

(46) Bae, Y. J.; Shimizu, D.; Schultz, J. D.; Kang, G.; Zhou, J.; Schatz, G. C.; Osuka, A.; Wasielewski, M. R. J. Phys. Chem. A 2020, 124, 8478.

(47) Austin, A.; Hestand, N. J.; McKendry, I. G.; Zhong, C.; Zhu, X.; Zdilla, M. J.; Spano, F. C.; Szarko, J. M. J. Phys. Chem. Lett. 2017, 8, 1118.

(48) Wehner, M.; Röhr, M. I. S.; Bühler, M.; Stepanenko, V.; Wagner, W.; Würthner, F. J. Am. Chem. Soc. 2019, 141, 6092

(49) (a) Hestand, N. J.; Spano, F. C. Acc. Chem. Res. 2017, 50, 341. (b) Hestand, N. J.; Spano, F. C. Chem. Rev. 2018, 118, 7069.

(50) (a) Smith, M. B.; Michl, J. Chem. Rev. 2010, 110, 6891. (b) Smith, M. B.; Michl, J. Annu. Rev. Phys. Chem. 2013, 64, 361. (c) Casanova, D. Chem. Rev. 2018, 118, 7164.

(51) Zimmerman, P. M.; Zhang, Z.; Musgrave, C. B. Nat. Chem. 2010, 2, 648.

(52) Walker, B. J.; Musser, A. J.; Beljonne, D.; Friend, R. H. Nat. Chem. 2013, 5, 1019.

(53) Mauck, C. M.; Hartnett, P. E.; Margulies, E. A.; Ma, L.; Miller, C. E.; Schatz, G. C.; Marks, T. J.; Wasielewski, M. R. J. Am. Chem. Soc. 2016, 138, 11749.

(54) Kolata, K.; Breuer, T.; Witte, G.; Chatterjee, S. ACS Nano 2014, 8, 7377.

(55) Stern, H. L.; Musser, A. J.; Gelinas, S.; Parkinson, P.; Herz, L. M.; Bruzek, M. J.; Anthony, J.; Friend, R. H.; Walker, B. J. Proc. Natl. Acad. Sci. U. S. A. 2015, 112, 7656.

(56) Korovina, N.; Das, S.; Nett, Z.; Feng, X.; Joy, J.; Haiges, R.; Krylov, A. I.; Bradforth, S. E.; Thompson, M. E. J. Am. Chem. Soc. 2016, 138, 617.

(57) Feng, X.; Krylov, A. I. Phys. Chem. Chem. Phys. 2016, 18, 7751.

(58) Dover, C. B.; Gallaher, J. K.; Frazer, L.; Tapping, P. C.; Petty, A. J.; Crossley, M. J.; Anthony, J. E.; Kee, T. W.; Schmidt, T. W. Nat. Chem. 2018, 10, 305.

(59) Eaton, S. W.; Shoer, L. E.; Karlen, S. D.; Dyar, S. M.; Margulies, E. A.; Veldkamp, B. S.; Ramanan, C.; Hartzler, D. A.; Savikhin, S.; 
Marks, T. J.; Wasielewski, M. R. J. Am. Chem. Soc. 2013, 135, 14701.

(60) Hong, Y.; Kim, J.; Kim, W.; Kaufmann, C.; Kim, H.; Würthner, F.; Kim, D. J. Am. Chem. Soc. 2020, 142, 7845.

(61) (a) Margulies, E. A.; Miller, C. E.; Wu, Y.; Ma, L.; Schatz, G. C.; Young, R. M.; Wasielewski, M. R. Nat. Chem. 2016, 8, 1120. (b) Margulies, E. A.; Logsdon, J. L.; Miller, C. E.; Ma, L.; Simonoff, E.; Young, R. M.; Schatz, G. C.; Wasielewski, M. R. J. Am. Chem. Soc. 2017, 139, 663.

(62) Bartynski, A. N.; Gruber, M.; Das, S.; Rangan, S.; Mollinger, S.; Trinh, C.; Bradforth, S. E.; Vandewal, K.; Salleo, A.; Bartynski, R. A.; Bruetting, W.; Thompson, M. E. J. Am. Chem. Soc. 2015, 137, 5397.

(63) Vauthey, E. ChemPhysChem. 2012, 13, 2001.

(64) (a) Jia, Y.; DiMagno, T. J.; Chan, C. K.; Wang, Z.; Popov, M. S.; Du, M.; Hanson, D. K.; Schiffer, M.; Norris, J. R.; Fleming, G. R. J. Phys. Chem. 1993, 97, 13180. (b) Laporte, L. L.; Palaniappan, V.; Davis, D. G.; Kirmaier, C.; Schenck, C. C.; Holten, D.; Bocian, D. F. J. Phys. Chem. 1996, 100, 17696. (c) Moore, L. J.; Zhou, H.; Boxer, S. G. Biochemistry 1999, 38, 11949.

(65) (a) Wu, Y.; Young, R. M.; Frasconi, M.; Schneebeli, S. T.; Spenst, P.; Gardner, D. M.; Brown, K. E.; Würthner, F.; Stoddart, J. F.; Wasielewski, M. R. J. Am. Chem. Soc. 2015, 137, 13236. (b) Spenst, P.; Young, R. M.; Wasielewski, M. R.; Würthner, F. Chem. Sci. 2016, 7, 5428.

(66) Sung, J.; Nowak-Krol, A.; Schlosser, F.; Fimmel, B.; Kim, W.; Kim, D.; Würthner, F. J. Am. Chem. Soc. 2016, 138, 9029.

(67) Ramirez, C. E.; Chen, S.; Powers-Riggs, N. E.; Schlesinger, I.; Young, R. M.; Wasielewski, M. R. J. Am. Chem. Soc. 2020, 142, 18243.

(68) (a) Markovic, V.; Villamaina, D.; Barabanov, I.; Lawson Daku, L. M.; Vauthey, E. Angew. Chem. Int. Ed. 2011, 50, 7596. (b) Cook, R. E.; Phelan, B. T.; Kamire, R. J.; Majewski, M. B.; Young, R. M.; Wasielewski, M. R. J. Phys. Chem. A 2017, 121, 1607. (c) Dereka, B.; Vauthey, E. J. Phys. Chem. Lett. 2017, 8, 3927. (d) Coleman, A. F.; Chen, M.; Zhou, J.; Shin, J. Y.; Wu, Y.; Young, R. M.; Wasielewski, M. R. J. Phys. Chem. C 2020, 124, 10408.

(69) Kim, W.; Nowak-Krol, A.; Hong, Y.; Schlosser, F.; Würthner, F.; Kim, D. J. Phys. Chem. Lett. 2019, 10, 1919.

(70) Schulze, T. F.; Schmidt, T. W. Energy Environ. Sci. 2015, 8, 103.

(71) Ye, C.; Gray, V.; Martensson, J.; Börjesson, K. J. Am. Chem. Soc. 2019, 141, 9578

(72) Kim, W.; Kim, T.; Kang, S.; Hong, Y.; Würthner, F.; Kim, D. Angew. Chem. Int. Ed. 2020, 132, 8571.

(73) (a) Tretiak, S.; Saxena, A.; Martin, R. L.; Bishop, A. R. Phys. Rev. Lett. 2002, 89, 097402. (b) Aggarwal, A. V.; Thiessen, A.; Idelson, A.; Kalle, D.; Würsch, D.; Stangl, T.; Steiner, F.; Jester, S.-S.; Vogelsang, J.; Höger, S.; Lupton, J. M. Nat. Chem. 2013, 5, 964.

(74) Stangl, T.; Wilhelm, P.; Schmitz, D.; Remmerssen, K.; Henzel, S.; Jester, S. S.; Höger, S.; Vogelsang, J.; Lupton, J. M. J. Phys. Chem. Lett. 2015, 6, 1321. 